\title{
DNA Damage Response Protein CHK2 Regulates Metabolism in Liver Cancer
}

Matteo Lulli', Laura Del Coco ${ }^{2}$, Tommaso Mello ${ }^{3}$, Caecilia Sukowati ${ }^{4}$, Stefania Madiai ${ }^{5}$, Laura Gragnani ${ }^{5}$, Paolo Forte ${ }^{3}$, Francesco Paolo Fanizzi ${ }^{2,6}$, Antonio Mazzocca ${ }^{7}$, Krista Rombouts ${ }^{8}$, Andrea Galli ${ }^{3}$, and Vinicio Carloni ${ }^{5}$

\section{ABSTRACT}

Defective mitosis with chromosome missegregation can have a dramatic effect on genome integrity by causing DNA damage, activation of the DNA damage response (DDR), and chromosomal instability. Although this is an energy-dependent process, mechanisms linking DDR to cellular metabolism are unknown. Here we show that checkpoint kinase 2 (CHK2), a central effector of DDR, regulates cellular energy production by affecting glycolysis and mitochondrial functions. Patients with hepatocellular carcinoma (HCC) had increased CHK2 mRNA in blood, which was associated with elevated tricarboxylic acid cycle (TCA) metabolites. CHK2 controlled expression of succinate dehydrogenase (SDH) and intervened with mitochondrial functions. DNA damage and CHK2 promoted SDH activity marked by increased succinate oxidation

\section{Introduction}

Abnormal chromosome number termed aneuploidy is an aspect of cancer cells, a state in which cells do not contain an exact multiple of the haploid DNA content (1). Aneuploidy is not synonymous with chromosomal instability (CIN); some tumors are stably aneuploid with a highly abnormal but fairly uniform karyotype. In other tumors, an increased rate of CIN generates diverse karyotypes within a tumor (2). CIN affects chromosome number and structure and is a characteristic of many cancer types including hepatocellular carcino$\mathrm{ma}$; it is also associated with the formation of extranuclear bodies that contain damaged chromosome fragments or whole chromosomes. Such micronuclei were identified in regenerative and dysplastic

'Department of Experimental and Clinical Biomedical Sciences "Mario Serio", General Pathology Unit, University of Florence, Florence, Italy. ${ }^{2}$ Dipartimento di Scienze e Tecnologie Biologiche ed Ambientali, University of Salento, Lecce, Italy. ${ }^{3}$ Department of Experimental and Clinical Biomedical Sciences "Mario Serio", Gastroenterology Unit, University of Florence, Florence, Italy. ${ }^{4}$ Fondazione Italiana Fegato, AREA Science Park, Trieste, Italy, Laboratory of Molecular Biology and DNA repair, Department of Medicine (DAME), University of Udine, Udine, Italy. ${ }^{5}$ Department of Experimental and Clinical Medicine, University of Florence, Florence, Italy. ${ }^{6}$ Consorzio Interuniversitario di Ricerca in Chimica dei Metalli nei Sistemi Biologici (CIRCMSB), Bari, Italy. ${ }^{7}$ Interdisciplinary Department of Medicine, University of Bari, School of Medicine, Bari, Italy. ${ }^{8}$ University College London (UCL) Institute for Liver \& Digestive Health, London, United Kingdom.

Note: Supplementary data for this article are available at Cancer Research Online (http://cancerres.aacrjournals.org/).

Corresponding Author: Vinicio Carloni, Department of Experimental and Clinical Medicine, University of Florence, Largo Brambilla 3, Florence I-50134. Phone: 39-55-2758091; E-mail: vinicio.carloni@unifi.it

Cancer Res 2021;81:2861-73

doi: 10.1158/0008-5472.CAN-20-3134

(C2021 American Association for Cancer Research. through the TCA cycle; this was confirmed in a transgenic model of HCC with elevated DNA damage. Mitochondrial analysis identified CHK2-controlled expression of SDH as key in sustaining reactive oxygen species production. Cells with DNA damage and elevated CHK2 relied significantly on glycolysis for ATP production due to dysfunctional mitochondria, which was abolished by CHK2 knockdown. This represents a vulnerability created by the DNA damage response that could be exploited for development of new therapies.

Significance: This study uncovers a link between a central effector of DNA damage response, CHK2, and cellular metabolism, revealing potential therapeutic strategies for targeting hepatocellular carcinoma.

nodules of the liver, indicating that CIN can be acquired already in early stage of hepatocarcinogenesis. CIN generates diverse karyotypes within a tumor thus yielding a heterogeneously tumor cell population that has the ability to undergo selective evolution in terms of drug resistance and tumor escape from the immune system surveillance (3-5). We now appreciate that defective mitoses with chromosome missegregation can have a dramatic effect on genome integrity by causing DNA damage and activation of DNA damage response proteins (DDR; refs. 6-8). The effects of DDR activation during mitosis have remained obscure; however, it has been recently recognized that activation of the DDR may be an insidious phenomenon in chromosomally unstable cancer due to the intrinsic and enduring level of DNA damage during mitosis (9). Cancer cells may have lost the fine tuning of DDR proteins, which links mitosis and DNA damage to numerical and structural chromosomal aberrations (10). In this context, how genome instability of cancer cells regulates the generation of energy and the role of DDR pathway in cancer cell metabolism is unknown. Here, we establish that checkpoint kinase 2 (CHK2), a central effector of DDR pathway, controls mitochondrial functions and glycolysis. The majority of patients with cancer that undergo curative therapy have extremely high recurrence rates including those treated with innovative immune checkpoint inhibitors. Therefore, an urgent need for new therapies is justified. The results of this study provide new findings on DNA damage/CIN and cellular metabolism that offer helpful diagnostic and therapeutic tools in the management of cancer.

\section{Materials and Methods}

\section{Cell cultures and antibodies}

HCT116 were analyzed by karyotyping (see Supplementary Fig. S1) and RNA sequencing (RNA-seq; see Fig. 6), Huh7 were previously characterized by karyotyping (7) and analyzed by RNA-seq. Human hepatocytes immortalized with TERT gene (HuS) were provided by Makoto Hijikata (Laboratory of Human Tumor Viruses, Institute of 
Virus Research, Kyoto University, Kyoto, Japan) and characterized by karyotyping (7) and RNA-seq. HCT116 and Huh7 were grown in DMEM supplemented with $10 \% \mathrm{FBS}, 1 \%$ penicillin/streptomycin, and $1 \%$ glutamine at $37^{\circ} \mathrm{C}$ and $95 \%$ humidity and $5 \% \mathrm{CO}_{2} . \mathrm{HuS}$ cells were cultured in DMEM supplemented with 5\% FBS, 5\% normal human serum from male type AB serum (Sigma-Aldrich), 1\% DMSO, $0.1 \mu \mathrm{L} / \mathrm{mL}$ insulin, $0.1 \mu \mathrm{L} / \mathrm{mL}$ hydrocortisone, and $2 \mathrm{ng} / \mathrm{mL}$ EGF (Calbiochem). Cells were routinely assayed for Mycoplasma contamination. Antibodies employed were anti-CHK2 (Cell Signaling Technology, \# 3440), anti-phospho-Histone H2AX (Ser139; Cell Signaling Technology, \#9718), anti-PKM2 (Cell Signaling Technology, \#4053), anti-SDHA (Cell Signaling Technology, \#11998), anti-CENPA (Abcam,\#13939), anti-PGK1 (Abcam, \#154613), anti-CHK2 (Abcam, \#47433), anti- $\beta$-actin (clone AC-15; Sigma-Aldrich). Secondary antibodies conjugated to Alexa Fluor 488 and 594 (Molecular Probes) were used in immunofluorescence analysis. Secondary antibodies used in immunoblot analysis were IRDye 800CW anti-rabbit (\#926-32210, Lycor Bioscience) and IRDye 800CW anti-mouse (\#926-32211, Lycor Bioscience). DNA was stained with Hoechst 33342 (ThermoFisher Scientific).

\section{Measurements of extracellular vesicle-derived CHK2 mRNA in human blood}

Blood samples from patients were obtained with written informed consent according to the procedures approved by the Institutional Review Board. After collection and centrifugation, serum was frozen at $-80^{\circ} \mathrm{C}$ for $\mathrm{CHK} 2$ and metabolomic analysis. Purification of total RNA from extracellular vesicles (EV), including mRNA, was performed by using the exoRNeasy Serum/Plasma Maxi Kit (Qiagen) according to the manufacturer's instructions. Total RNA was quantified using a Nanodrop 2000 UV-visible spectrophotometer. cDNA was prepared using $20-50 \mathrm{ng} / \mathrm{mL}$ total RNA by a RT-PCR using a High Capacity cDNA Reverse Transcription Kit (Applied Biosystems), according to the manufacturer's instructions. qPCR was performed on cDNA using TaqMan probes specific for CHK2 (Hs 01007282_m1, Thermo Fisher Scientific) and the human GAPDH FAM/MGB probe, qPCR was performed on a 7900 HT Fast Real-Time PCR System (Applied Biosystems). Fold changes in expression were calculated by the $\Delta \Delta C_{\mathrm{t}}$ method using GAPDH as an endogenous control for mRNA expression.

We considered patient samples $C H K 2$ negative, those samples with a value of $2^{-\Delta C_{\mathrm{t}}\left[C_{\mathrm{t}} \mathrm{CHK} 2-C_{\mathrm{t}} \mathrm{GAPDH}\right]} \leq$ to mean $2^{-\Delta C_{\mathrm{t}}}$ of healthy subjects.

\section{HBV-transgenic mouse model}

An HBV-transgenic mouse strain was used as a model of hepatocarcinogenesis $(11,12)$. Male C57BL/6J-TG(ALB1HBV)44BRI/J transgenic mice (TG) and its normal C57BL/6J (WT) were maintained at the animal facility of the University of Trieste (Trieste, Italy). At 15 months, the incidence and the size of the macroscopic tumoral masses were evident and most of the tumors were vascularized. In transgenic mice, the appearance of ground glass hepatocytes, clear cells, ballooning, and necrosis characterized the process. Moreover, nuclear alteration, cellular polymorphism, intracellular acidophilic bodies, Mallory bodies, and nucleoli were documented. The transgenic livers were also characterized by the presence of inflammation and activated resident immunologic cells, perivascular fibrosis, and several degree of steatosis. Furthermore, serum alanine aminotransferase (ALT) level was more than two times higher in transgenic mice compared with wild-type mice. Animal experimentation was carried out in accordance with the Guide for the Care and Use of Laboratory Animals. The protocol of animal study were approved by the ethical committee of the University of Trieste and by the responsible administration of the Ministry of Health (D.M. 57/2012-B). Liver tissues were collected during animal sacrifice at age of $3,6,9,12$, and $>15$ months for both TG and WT mice. Upon collection, tissues were immediately fixed in formalin and included in paraffin block. Paraffinated slices $(3.5 \mu \mathrm{m})$ were then subjected to hematoxylin and eosin for histologic analysis and immunostaining. PKM2 and SDHA expression were scored according to the percentage of hepatocytes $(n=100)$ or tumor cells $(n=100)$ stained positive for PKM2 or SDHA. Scoring included only cells with cytoplasmic staining intensity $3+$ at $10 \times$ and $25 \times$ magnifications.

\section{Retroviral infections}

The following shRNAs (OriGene Technologies) were delivered into HCT116, Huh7 cells by retroviral infection according to the manufacturer's instructions.TF320655 is a set of plasmids containing four shRNA constructs in retroviral vector (pRFP-C-RS) to knockdown human CHK2. TR30015 is a noneffective 29-mer scrambled shRNA used as control. Retroviral particles were generated by transient transfection of the Phoenix-amphotropic packaging cell line as described previously (13).

\section{Western blot analysis}

Standard procedures were used (7). Briefly, protein lysates were quantified by the Bradford method (Bio-Rad) and fifty micrograms of total protein were denaturated and separated on MiniProtean TGX precast gels (Bio-Rad).

\section{Cell-cycle analysis}

HuS0gen, HuS30gen, HCT116shCTL, HCT116shCHK2, Huh7shCTL, and Huh7shCHK2 cells were trypsinized, pelleted, and resuspended in a solution containing $50 \mu \mathrm{g} / \mathrm{mL}$ propidium iodide, $0.1 \% \mathrm{w} / \mathrm{v}$ trisodium citrate, and $0.1 \% \mathrm{NP} 40$. Samples were then incubated for 30 minutes at $4{ }^{\circ} \mathrm{C}$ in the dark and nuclei analyzed with a FACS Canto II flow cytometer (Becton Dickinson).

\section{NMR measurements}

Cells were cultured in complete DMEM or labeled in media supplemented with $10 \mathrm{mmol} / \mathrm{L}$ of $\left[\mathrm{U}_{-}{ }^{13} \mathrm{C}_{6}\right.$ ] glucose for ${ }^{13} \mathrm{C}$ tracer experiments (Cambridge Isotope Laboratories). The concentration is based on the standard DMEM formulation. At the time of collection, the cells were washed twice with ice cold PBS and lysed in ice-cold PBS by sonication. After removing the insoluble particles by centrifugation, the supernatants were immediately frozen at $-80^{\circ} \mathrm{C}$. Both cell lysates and culture media (500 $\mu \mathrm{L}$ samples) were added to $100 \mu \mathrm{L} \mathrm{D}_{2} \mathrm{O}$ buffer $\left(0.1 \mathrm{M} \mathrm{K}_{2} \mathrm{HPO}_{4}, 0.5 \mathrm{mmol} / \mathrm{L}\right.$ DSS and 2 $\mathrm{mmol} / \mathrm{L}$ sodium azide, $\mathrm{pH} 7.4)$ and transferred in $5-\mathrm{mm}$ outer diameter NMR tubes $(14,15,16)$.

\section{$\mathrm{NAD}^{+} / \mathrm{NADH}$ and GSH measurements}

Cells were plated at $1.0 \times 10^{6}$ cells $/ \mathrm{mL}$ in $10-\mathrm{cm}$ non-cell culturetreated dishes $(10 \mathrm{~mL} / \mathrm{dish})$ and treated as required. $\mathrm{NAD}^{+} / \mathrm{NADH}$ and GSH were assayed using a quantification colorimetric kit (BioVision) according to the manufacturer's instructions.

\section{ATP levels dynamics measured by a fluorescence resonance energy transfer-biosensor}

Free ATP levels were quantified by using a fluorescence resonance energy transfer (FRET)-based indicator, namely ATeam 1.03 (AT1.03; ref. 17). This sensor is comprised of the $\varepsilon$ subunit of the 
bacterial Fo F1-ATP synthase sandwiched by cyan fluorescent protein (CFP) and yellow fluorescent protein (YFP) variants. Cells were plated on a glass-bottom dish and transfected with plasmid coding AT1.03 cDNA by using Lipofectamine 2000 transfection reagent. Forty-eight hours after transfection, cells (HuSOgen, HuS30gen, HCT116shCTRL, HCT116shCHK2, Huh7shCTRL, Huh7shCHK2) cultured in glucose-, glutamine-, and pyruvatefree DMEM were subjected to imaging. Preliminary experiments were done to define the dependence of cells studied on glucose and glutamine. All the cell lines evaluated were strongly dependent on glucose metabolism for proliferation and ATP production. Furthermore, we added 2-DG to block glucose metabolism derived from intracellular glycogen stores. Cells were maintained in an incubation chamber that was placed on the temperature-controlled $\left(37^{\circ} \mathrm{C}\right)$ stage of Nikon Eclipse TE2000-U (Nikon) inverted confocal microscope 405-nm laser source and 450/35 (for CFP) or 515/30 (for YFP) emission filters were used for dual-emission ratio imaging of AT1.03. The collected images were analyzed by ImageJ software; in particular, the YFP/CFP emission ratio was calculated by dividing pixel by-pixel a YFP image with a CFP image. We monitored the dynamics of ATP levels in living cells after the addition of 2-DG. In immunofluorescence experiments, colocalization was quantified using ImageJ with Pearson correlation coefficient (Pearson $r$ ), which ranges between 1 and -1 . A value of 1 represents perfect correlation, 0 means no correlation and -1 represents perfect negative correlation.

\section{ROS quantification}

Cells were incubated with $5 \mathrm{mmol} / \mathrm{L}$ 29,79-dichlorodihydrofluorescein diacetate (DCFDA, Invitrogen) for 30 minutes. Excess DCFDA was removed by washing the cells twice with PBS, and labeled cells were then trypsinized, rinsed, and resuspended in PBS. Oxidation of DCFDA to the highly fluorescent 29,79-dichloro-fluorescein (DCF) is proportionate to ROS generation and was analyzed by flow cytometry.

Mitochondrial membrane potential

Cells were plated at $0.1 \times 10^{6}$ cells $/ \mathrm{mL}$ in DMEM containing $10 \%$ FBS. TMRM cell-permeable fluorescent dye was either added before or after stimulation depending on the length and nature of treatment. In the case of inhibitor-treated cells, media were removed and replaced with TMRM-containing media $(100 \mathrm{nmol} / \mathrm{L})$ and incubated at $37^{\circ} \mathrm{C}$ in the dark for 30 minutes. Oligomycin or CCCP was added after the addition of TMRM for 1 hour or 2 minutes, respectively. The cellpermeable MitoTracker Green FM (Molecular Probes) was employed to label mitochondria. Cells were imaged on a confocal microscope with an excitation laser of $550 \mathrm{~nm}$ and detection set for 560-650 nm using a 40x oil-objective lens. A number of images were taken for each treatment

\section{Transcriptomics analysis}

RNA was isolated from HuS0gen, HuS30gen, HCT116shCTL, HCT116shCHK2, Huh7shCTL, and Huh7shCHK2 using the RNeasy Mini kit (QIAGEN) according to the manufacturer's instructions and quantified using a Nanodrop 2000 UV-visible spectrophotometer. RNA integrity was confirmed using Agilent's 2200 Tape station. Samples were processed using the KAPA mRNA HyperPrep Kit ( $\mathrm{p} /$ nKK8580) according to manufacturer's instructions at the UCL Genomics core facility, University College London (London, United Kingdom). Differential gene expression in the cells HuSOgen versus HuS30gen, HCT116shCTL versus HCT116shCHK2, and Huh7shCTL versus Huh7shCHK2 was compared ( $n=1$ biological sample). A list of genes with $\log _{2}$ FoldChange, dispGeneEst, and $P$ value of 0.05 was obtained $(18,19)$.

\section{Statistical analysis}

The data are presented mean \pm SD from at least three independent experiments with similar results. All presented immunofluorescence images are representative images from three representative experiments. For the quantification of immunofluorescence images, the number of cells used for each representative experiment is indicated and $P$ values between two groups were determined using unpaired $t$ tests. For results encompassing multiple groups, one-way ANOVA was employed. For other experiments statistical significance was determined using an unequal variance $t$ test. $P$ values of less than 0.05 were considered significant. Plots and statistical analysis were constructed using software GraphPad Prism Version 7.0.

Detailed methods are provided in the Supplementary Methods section.

\section{Results}

Circulating extracellular vesicles-CHK2 mRNA and metabolomic profile in HCC patients

We previously reported that the expression levels of CHK2 are increased in human HCC (7). In this study, we explored the feasibility of measuring $\mathrm{CHK} 2 \mathrm{mRNA}$ in the blood to investigate the presence of DNA damage/chromosomal instability in liver patients. To this end, we employed sera from 22 patients with HCC, 14 patients with liver cirrhosis, and 20 healthy subjects, Supplementary Table S1. To avoid the possibility that the presence of CHK2 mRNA may be linked to necrotic/apoptotic phenomena or mRNA been degraded by blood RNases, we extracted the extracellular vesicleassociated total RNA from the blood. We found a significant increase of $C H K 2$ mRNA derived from HCC compared with healthy subjects (Fig. 1A). Remarkably, CHK2 mRNA was increased in 7 of 14 patients with cirrhosis, thus suggesting the presence of DNA damage in hyperplastic/dysplastic nodules. Next, we investigated the metabolic changes occurring in patients with $\mathrm{HCC} /$ cirrhosis with increased CHK2 mRNA expression. We therefore performed a metabolomic analysis by using ${ }^{1} \mathrm{H}$-NMR spectroscopy to characterize and compare the serum fingerprints of patients (20). The results showed differences in the metabolomic profile of $C H K 2$-positive patients with significantly increase of citrate, fumarate, and formate in comparison with CHK2negative patients (Fig. 1B). In addition, lactate levels were significantly increased in CHK2-positive patients compared with CHK2-negative patients (Fig. 1B). Metabolomic data revealed an association of $\mathrm{CHK} 2$ with glycolysis and TCA cycle. In particular, we investigated the link of CHK2 with the remarkable increase of fumarate compared with other TCA cycle metabolites, and glycolytic intermediates as well. We focused our attention on PKM2, which is a rate-limiting glycolytic enzyme, and the M2 isoform of PK predominantly expressed in proliferating cells and tumors $(21,22)$. Succinate dehydrogenase (SDH) is a highly conserved mitochondrial heterotetrameric (SDHA, SDHB, SDHC, SDHD) and functions as complex II of the electron transport chain (ETC), catalyzing the oxidation of succinate to fumarate. In recent years, several studies have highlighted the role of the $\mathrm{SDH}$, succinate, fumarate in biological processes other than metabolism. For this reason, SDH has now been involved in tumorigenesis and both succinate and fumarate are considered oncometabolites (23). To clarify the role of glycolysis and mitochondrial activity in dysplastic/neoplastic tissues marked with DNA damage, we employed a transgenic model of HCC that overproduces the HBV 
Lulli et al.

A

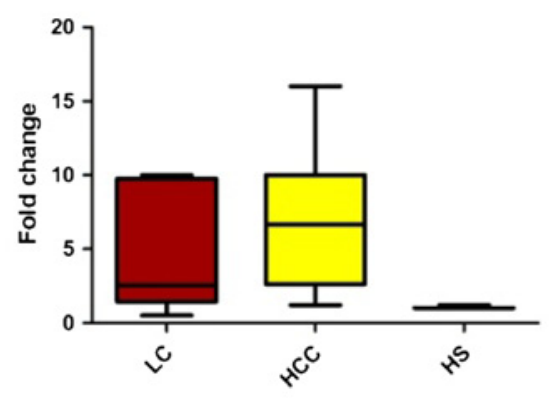

WT $15 \mathrm{~m}$
B

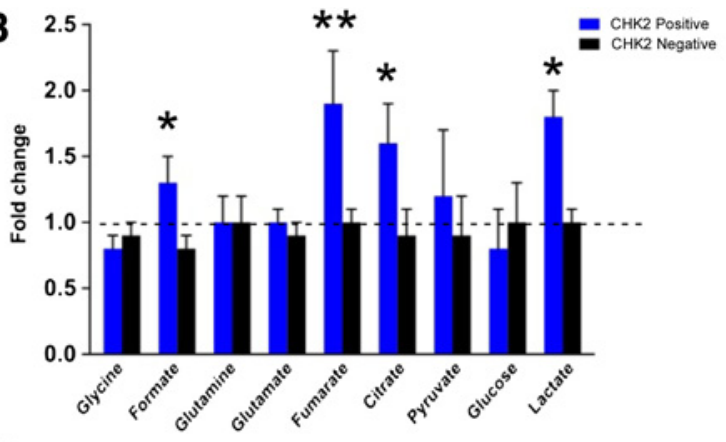

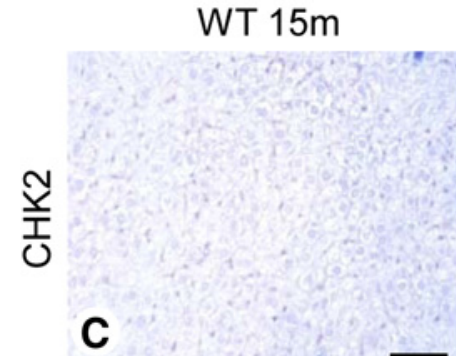
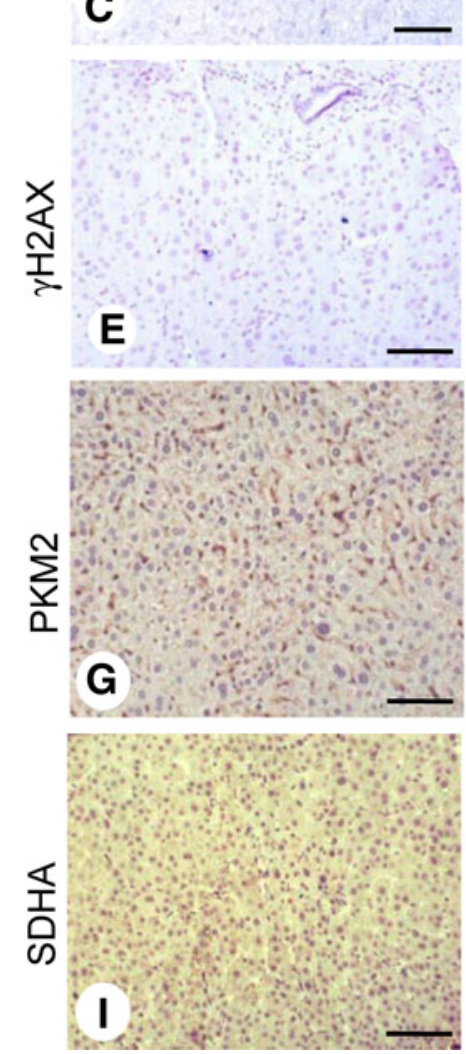

TG $15 \mathrm{~m}$
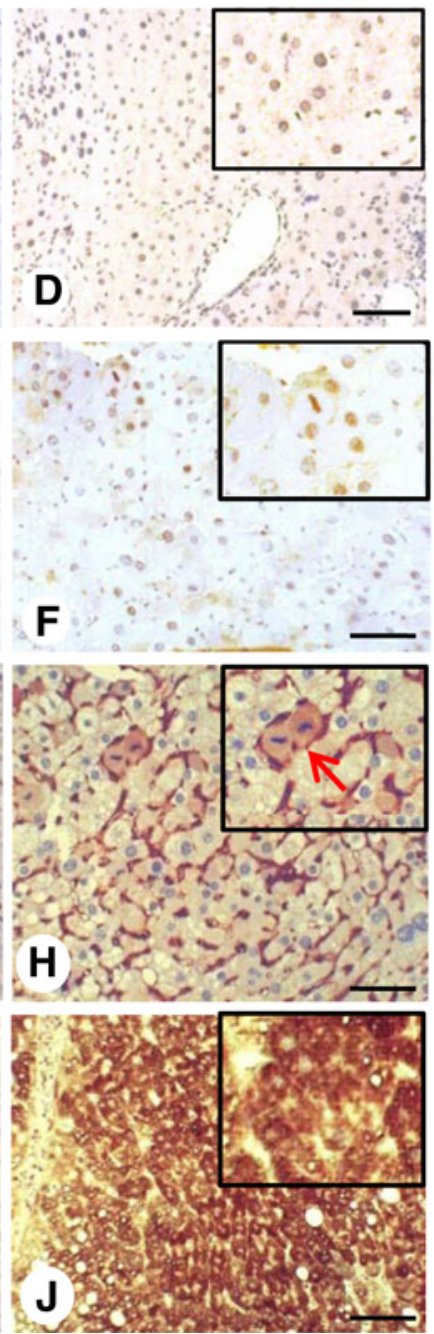

K
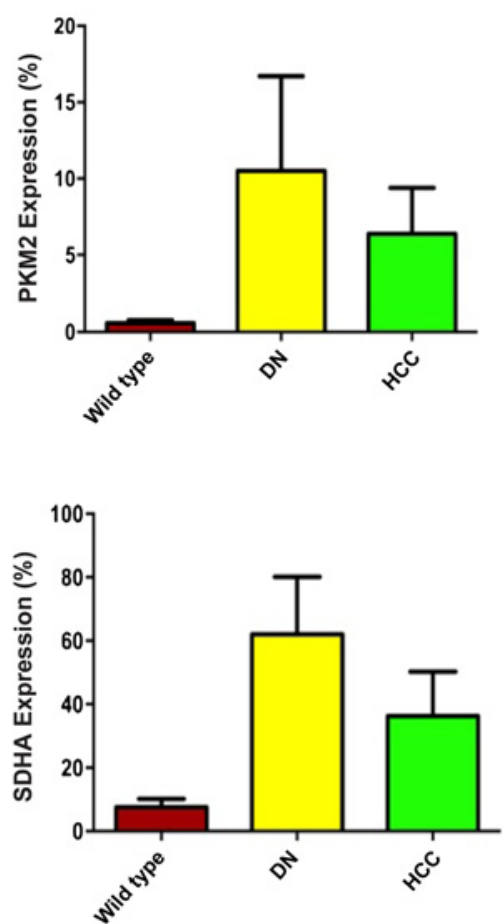

Figure 1.

CHK2 and metabolomic profile in patients with $\mathrm{HCC}$ and IHC detection of CHK2, PKM2, and SDHA in a transgenic model of HCC. A, Box plots of fold change values for the CHK2 mRNA in patients with liver cirrhosis (LC), HCC, and healthy subjects (HS) serum samples. Data were analyzed by using one-way ANOVA ( $P=0.0153 ; \mathrm{F}=$ 5.133). B, Comparison of metabolite serum levels between $C H K 2$-positive patients and CHK2-negative patients, and all the values were normalized and displayed relative to metabolite levels of healthy subjects, which were arbitrarily set at $1 .{ }^{*}, P<0.05 ;{ }^{* *}, P<0.01$, by two-tailed $t$ test. C-J, Representative image of livers of 15-month-old wild-type mice $(n=10)$ used as control (left) and 15-month-old HBV transgenic mice $(n=20$; right). Scale bar, $1 \mathrm{~mm}$. C and D, CHK2 immunoreactivity. See nuclear staining in the right panels. $\mathbf{E}$ and $\mathbf{F}$, Interphase and metaphase nuclear $\gamma \mathrm{H} 2 \mathrm{AX}$ immunoreactivity in HBV transgenic mice (right). $\mathbf{G}$ and $\mathbf{H}$, PKM2 protein expression. Wild-type mice express PKM2 in hepatic sinusoids. HBV transgenic mice express PKM2 in hepatic sinusoids and hepatocytes (red arrow, metaphase of mitotic cells). I and J, Liver tissue of wild-type mice is characterized by a moderate, diffuse cytoplasmic SDHA staining (left). SDHA immunoreactivity in liver of HBV transgenic mice shows a clear and strong cytoplasmic staining (right). K, PKM2 and SDHA expression was scored according to the percentage of hepatocytes ( $n=$ $100)$ or tumor cells $(n=100)$ stained positive for PKM2 or SDHA. Scoring included only cells with cytoplasmic staining intensity $3+$ at $\times 10$ and $\times 25$ magnifications. 
CHK2 as a Sensor of Cellular Metabolic Requirements

large envelope polypeptide, namely C57BL/6J-TG(ALB1-HBV) 44BRI/J. In this transgenic model, mice developed hepatocyte damage and inflammation early, generating dysplastic nodules by the age of 9 months with macroscopic HCC nodules by the age of $\geq 12$ months (12). In this setting the expression of CHK2, phosphohistone $\mathrm{H} 2 \mathrm{AX}(\gamma \mathrm{H} 2 \mathrm{AX}$, a marker of DNA double-stranded breaks), PKM2 and SDHA catalytic subunit was evaluated by IHC. We detected the presence of numerous mitoses in dysplastic/neoplastic lesions of 15-month-old transgenic mice, where hepatocytes exhibited nuclear upregulation of CHK2 (Fig. 1C and D) and $\gamma \mathrm{H} 2 \mathrm{AX}$ (Fig. 1E and F) in comparison with wild-type mice, thus confirming the presence of DNA damage (24). Liver tissue of wild-type mice showed PKM2 expression exclusively localized in the cells of hepatic sinusoids (Fig. 1G). Conversely, dysplastic/neoplastic tissue showed high expression of PKM2 protein in both hepatic sinusoids and cytoplasm of hepatocytes (Fig. 1H). SDHA was strongly overexpressed in

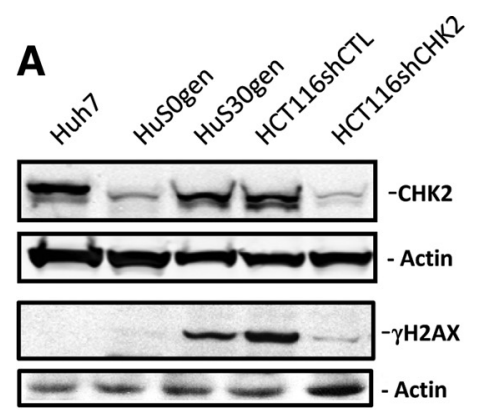

B

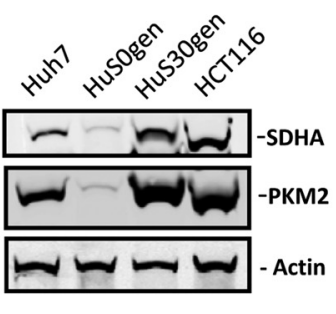

C

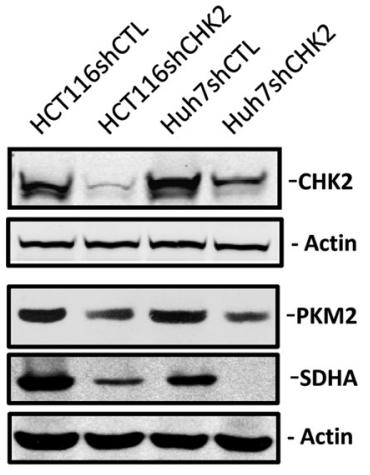
HuS30gen

D

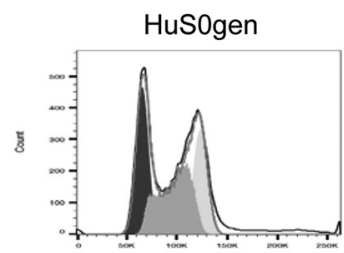

要

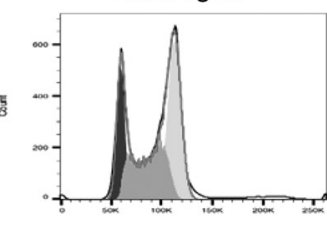

HCT116shCTL

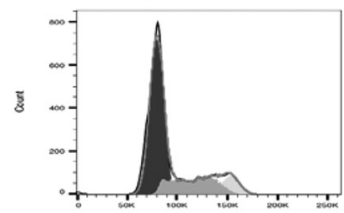

HCT116shCHK2

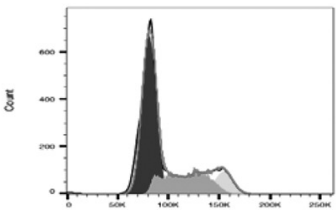

Huh7shCTL

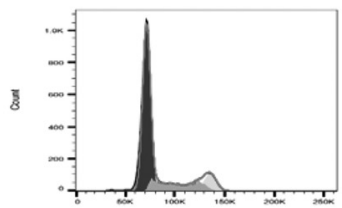

Huh7shCHK2

s
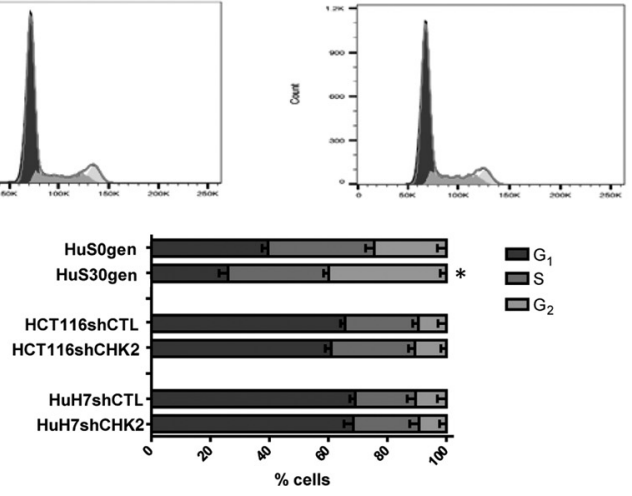

$\square \mathrm{s}$

$\square \mathrm{G}_{2}$

Figure 2.

Loss of CHK2 does not impair normal growth. A, Representative immunoblot out of three independent experiments demonstrating the protein levels of $\mathrm{CHK} 2$ and $\gamma \mathrm{H} 2 \mathrm{AX}$ in cultured HCT116, Huh7, HuSOgen cells, and HuSOgen cells after 30 generations in culture (HuS30gen); HCT116 cells were retrovirally transduced either with a control shRNA (shCTL) or shRNA targeting CHK2 (shCHK2). B, Representative immunoblot out of three independent experiments showing the PKM2 and SDHA protein expression in cultured Huh7 and HCT116 cells, or in HuSOgen and HuS30gen cells. C, HCT116 and Huh7 cells were retrovirally transduced either with a scrambled shRNA (shCTL) or shRNA targeting CHK2. The protein levels of CHK2, PKM2, and SDHA were determined by immunoblotting and a representative example is shown. D, Asynchronously growing HCT116, Huh7, CHK2-deficient HCT116, and Huh7 cells were subjected to FACS analyses. Typical FACS profiles show normal growth in parental cells and in CHK2-deficient cells. HuS30gen cells display a delay in generating cells with a $2 \mathrm{~N}$ DNA content compared with HuSOgen. 
dysplastic/neoplastic tissue of transgenic mice compared with the agematched wild type mice (Fig. 1I-K).

\section{Glycolytic enzymes PGK1 and PKM2 compartmentalize ATP production colocalizing with mitotic spindle components}

To gain further mechanistic insight into the role of DNA damage and CHK2 in glycolysis and oxidative metabolism an analysis in vitro was conducted. We used HuS (human hepatocytes cell line immortalized with telomerase reverse transcriptase (hTERT), and Huh7 cells. Huh7 is a chromosomally stable HCC cell line. HuS cells just established in culture (0 generation), hereafter referred to as HuSOgen, showed a karyotype with a distribution of 46 chromosomes. On the contrary, HuS cells maintained in culture and after 30 consecutive generations, hereafter referred to as HuS30gen, showed a distribution near triploid, with many structural chromosomal defects (7). We also generated a chromosomally unstable version of the colon carcinoma HCT116 cell line by continuously culturing cells, hereafter referred to as HCT116. These cells exhibited a distribution near-diploid with many structural chromosomal rearrangements as shown in Supplementary Fig. S1A and S1B. To evaluate the establishment of DNA damage, we tested the expression and localization of $\gamma \mathrm{H} 2 \mathrm{AX}$ and CHK2 $(25,26)$. As shown in Fig. 2A, HuS30gen showed higher levels of $\gamma \mathrm{H} 2 \mathrm{AX}$ and CHK2 in comparison with HuSOgen. Several foci of DNA damage were evident in HuS30gen cells with lagging chromosomes and in HCT116 (Supplementary Fig. S2A and S2B). Silencing of CHK2 in HCT116 cells (HCT116shCHK2) determined the reduction of $\gamma \mathrm{H} 2 \mathrm{AX}$ and CHK2 levels. In contrast, HuSOgen and Huh7 showed low levels of $\gamma \mathrm{H} 2 \mathrm{AX}$ and $\mathrm{CHK} 2$ (Fig. 2A). Next, we examined the expression levels of PKM2 and SDHA in HCT116, Huh7, HuS0gen, HuS30gen, and HCT116 cells. HCT116 cells revealed high levels of PKM2 and SDHA as well as HuS30gen showed increased SDHA and PKM2 levels in comparison with HuS0gen (Fig. 2B). Furthermore, CHK2-depleted HCT116 and Huh7 (HCT116shCHK2 and Huh7shCHK2, respectively) showed a marked reduction of both SDHA and PKM2 levels, compared with their controls (HCT116shCTL and Huh7shCTL; Fig. 2C). To uncover possible defects in mitosis induced by loss of CHK2, we performed cell-cycle analysis. No modification of cell cycle was observed in CHK2-depleted HCT116 and Huh7 cells. HuS30gen showed a delay in mitosis progression in comparison with HuSOgen (Fig. 2D). We hypothesized that under conditions of DNA damage/CHK2 expression, glycolytic ATP-producing enzymes such as PKM2 and phosphoglycerate kinase 1 (PGK1), the first ATP-generating enzyme that catalyzes the transfer of the high-energy phosphate from the 1 position of 1,3-diphosphoglycerate to ADP, redistribute from a diffuse localization in the cytoplasm to a localization in the mitotic spindle (27). Our hypothesis was verified by immunofluorescence experiments, indeed PKM2 and PGK1 colocalized with CENP-A, a constitutive component of kinetochores and CHK2 expression was required (Fig. 3A and C; Supplementary Fig. S3A and S3B). The most interesting aspect was that, in both HuS30gen and HCT116 cells, PGK1 and PKM2 colocalized with CHK2 (Supplementary Fig. S4A-S4D) suggesting the ad hoc formation of a glycolysis compartment able to maintain effective local levels of ATP. This result was confirmed also in living HuS30gen cells, where we verified colocalization of RFP-CHK2 with EGFP-PKM2 (Fig. 3D).

\section{DDR activation promotes a defective ATP mitochondrial production}

To assess the relationship between CHK2 expression, the glycolytic pathway and oxidative metabolism, we measured glycolysis (through measurements of the extracellular acidification rate, ECAR) and oxidative metabolism (oxygen consumption rate, OCR) by using Seahorse extracellular flux (XF-96) analyzer. HuS30gen and HCT116shCTL exhibited a significant increase of ECAR (Fig. $4 \mathrm{~A}$ and $\mathrm{B}$ ) and $\mathrm{O}_{2}$ consumption (Fig. 4D and E) compared with HuS0gen and HCT116shCHK2. Interestingly, Huh7 cells, after knockdown of $\mathrm{CHK} 2$, did not exhibit significant modifications in metabolic activity (Fig. 4C and F). Taken together, these data indicate that cells with DDR activation are characterized by increased glycolysis and oxidative metabolism.

ATP is the energy currency utilized by organisms in normal conditions and by cancer cells (28). To address the role of DNA damage/CHK2, glycolysis, and oxidative metabolism in the generation of ATP, we measured intracellular ATP in single living cells by using a FRET-based biosensor, namely ATeam. Our results showed that HuS30gen and HCT116shCTL cells significantly rely on glycolysis for the production of ATP. In addition, these cells exhibited an ostensibly defective function of mitochondria in the production of ATP. This impairment was rescued by gene knockdown of CHK2; again, Huh7 cells did not exhibit significant CHK2-depentent ATP production (Fig. 4G-I; Supplementary Fig. S5A-S5D).

\section{CHK2 controls glycolysis and TCA cycle intermediates production}

To further corroborate these findings, we performed steadystate metabolomic profiling by ${ }^{1} \mathrm{H}$ NMR spectroscopy of HuSOgen, HuS30gen, HCT116shCTL, HCT116shCHK2, Huh7shCTL, and Huh7shCHK2. As shown in Fig. 5A, HuSOgen and HCT116shCHK2 cells, showed a decreased intracellular lactate production, compared with HuS30gen and HCT116shCTL. Furthermore, HuSOgen and HCT116shCHK2 exhibited reduced levels of fumarate, a product of succinate oxidation. Interestingly, we observed that intracellular levels of aspartate and glutamate were significantly abundant in HuSOgen while HCT116shCHK2 showed a significant increase of only glutamate (Fig. 5B). Of note, these effects were inconsistent in CHK2depleted Huh7. To further elucidate the effect of CHK2 activity on glucose metabolism, we used $\left[\mathrm{U}_{-}{ }^{13} \mathrm{C}_{6}\right.$ ] glucose to trace the amount of ${ }^{13} \mathrm{C}$-alanine and ${ }^{13} \mathrm{C}$-lactate (in both medium and cell lysates (Supplementary Tables S2 and S3). HCT116shCTL and HuS30gen cell lysates exhibited an increased concentration of ${ }^{13} \mathrm{C}$-lactate. In particular, the enrichment percentage of ${ }^{13} \mathrm{C}$-lactate directly measured in the ${ }^{1} \mathrm{H}$ NMR spectra resulted of 25.13 and $24.49 \%$ for HCT116shCTL and HuS30gen, respectively (Supplementary Table S3). In addition, HCT116shCHK2 showed a significant higher concentration of ${ }^{13} \mathrm{C}$-alanine and the glycolytic intermediate phosphoenolpyruvate $\left({ }^{13} \mathrm{C}\right.$-PEP; Fig. 6A and B; Supplementary Figs. S6A-S66C and S7A-S7C). Moreover, we observed that the glucose oxidation via the TCA cycle in HCT116shCTL and HuS30gen produced increased concentration of ${ }^{13} \mathrm{C}$-fumarate, simultaneously high levels of ${ }^{13} \mathrm{C}$-glutamate in HCT116shCHK2 cell lysates were measured. Glutamate was significantly labeled at the $\mathrm{C} 4$ position and to a lesser extent at the $\mathrm{C} 2$ and $\mathrm{C} 3$ positions (Supplementary Fig. S6B). There are two ways of labeling from $\left[\mathrm{U}_{-}{ }^{13} \mathrm{C}_{6}\right]$ glucose into glutamate. One way involves the pyruvate dehydrogenase $(\mathrm{PDH})$ enzyme, which leads to the production of ${ }^{13} \mathrm{C}-4,5$-glutamate. The other way utilizes the anaplerotic reaction of pyruvate carboxylase to produce oxaloacetate directly from pyruvate, which generates ${ }^{13} \mathrm{C}-2,3$-glutamate. The presence of ${ }^{13} \mathrm{C}$-2,3-glutamate was consistent with activity of pyruvate carboxylase.

Next, we measured the glucose consumption of stable Huh7cells lacking significant levels of DNA damage. Huh7shCHK2 cells showed 
A
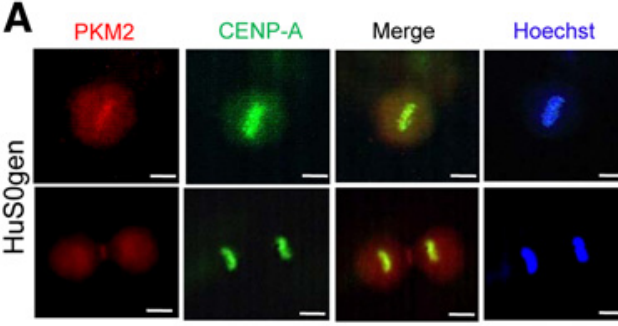

B
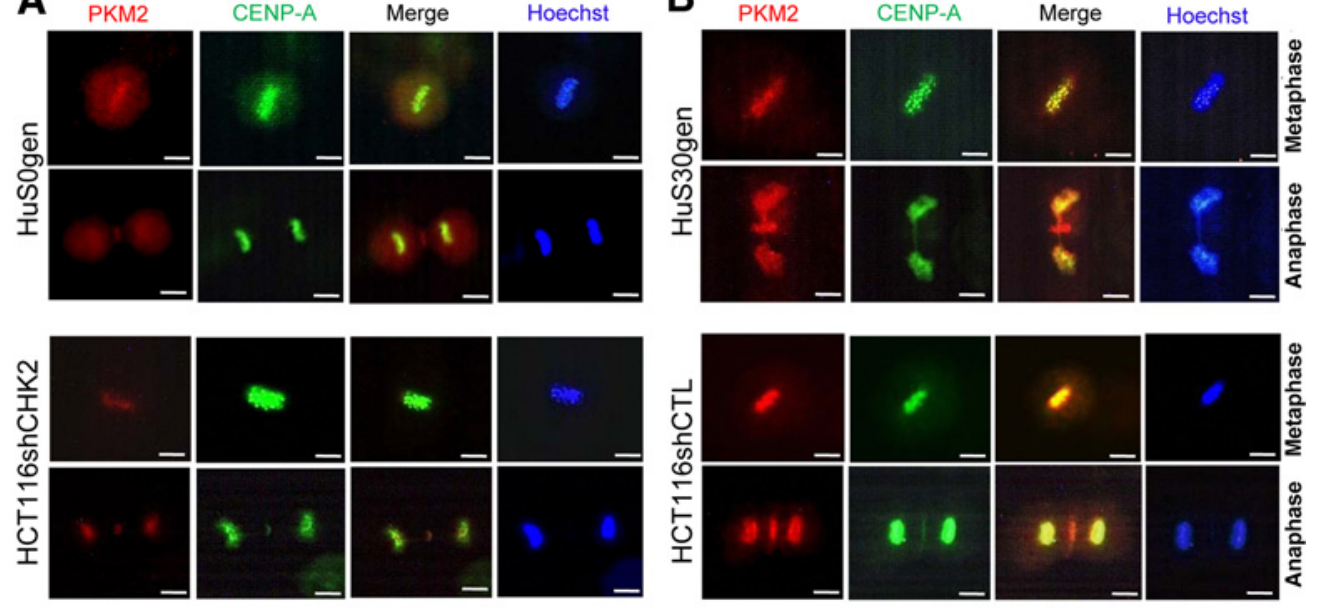

C

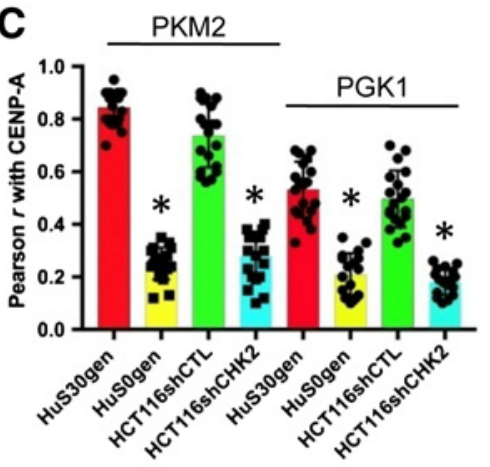

D
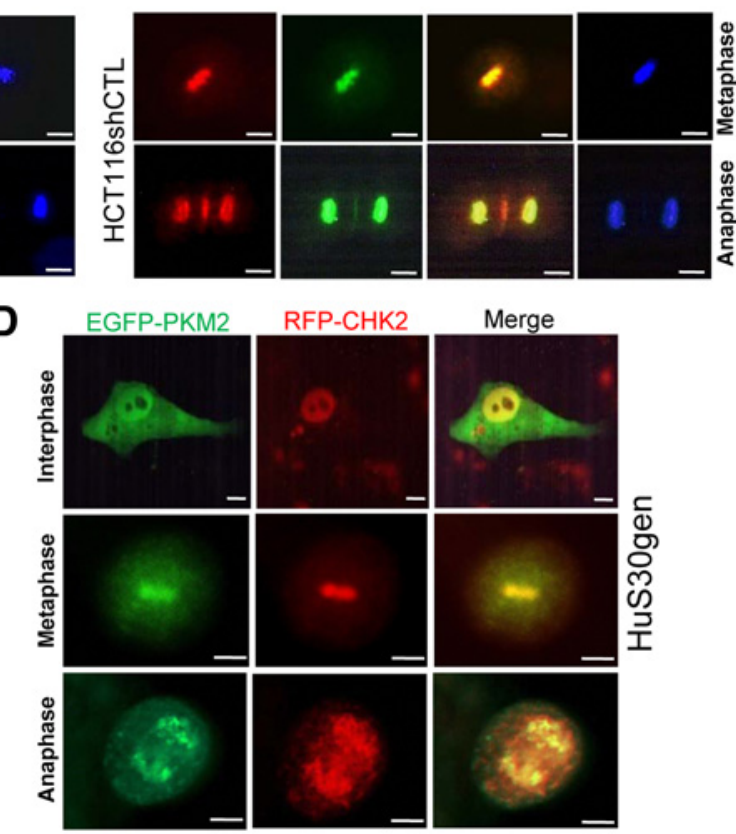

Figure 3.

Loss of CHK2 impairs colocalization of PKM2 with kinetochore component CENP-A. A and B, HuSOgen, HCT116shCHK2 and HuS30gen, HCT116shCTL cells were synchronized by thymidine treatment and then released into medium. Bipolar spindle assembly and chromosome alignment were monitored by immunofluorescence and typical examples are given. CENP-A (green), PKM2 (red), and chromosomes were stained with Hoechst 33342 (blue). C, Colocalization of PKM2 and PGK1 with CENP-A (see also Supplementary Fig. S3) was quantified using Pearson $r$. Data are mean \pm SD; $n=20$ cells from representative experiments. * $P<0.0001$. D, Images of PKM2 colocalization with CHK2 in living cells. HuS30gen were cotransfected with EGFP-tagged PKM2 (green) and RFP-tagged CHK2 (red); examples of colocalization in living cells are given. Scale bar, $10 \mu \mathrm{m}$.

an increased intracellular concentration of ${ }^{13} \mathrm{C}$-glucose and ${ }^{13} \mathrm{C}$-lactate (Fig. 6C). Furthermore, Huh7shCHK2 exhibited a raised glucose consumption up to $70 \%$, with increased ${ }^{13} \mathrm{C}$-lactate secretion (92.95\%; Supplementary Table S2). These results revealed a different function and specificity of CHK2 in HuS and HCT116 in comparison with Huh7 cells. To gain further insight into how $\mathrm{CHK} 2$ regulates cellular metabolism, we determined the effects of $\mathrm{CHK} 2$ silencing on the HCT116 and Huh7cells, by performing RNA-seq analysis (29). We focused on sets of genes that were related to the glycolytic processes, pentose phosphate pathway, TCA cycle, one-carbon unit metabolism, glutathione metabolism, and antioxidant pathways (Fig. 6D-F). Of particular relevance was that TCA-linked genes were for the most part downregulated in HCT116shCHK2 and reciprocally upregulated in Huh7shCHK2 (Fig. 6E and F). Furthermore, we evaluated the gene expression in HuSOgen and Hus30gen, confirming that the accumulating levels of DNA damage/CHK2 were able to control important pathways of cell metabolism such as glycolysis, pentose phosphate pathway, and TCA cycle (Fig. 6D). Overall, the metabolites changes were highly concordant with the gene expression changes from the RNA-seq profiles (Supplementary Fig. S8).

\section{Increased succinate oxidation induces the generation of mitochondrial ROS}

Next, we further investigated how CHK2 could affect mitochondrial functions. To this end, we took advantage of the previously obtained results regarding $\mathrm{O}_{2}$ consumption and ATP measurements. We observed that HuS30gen and HCT116shCTL cells displayed a higher OCR in comparison with HuSOgen and HCT116shCHK2 (Fig. 4D and E). However, the ability to produce ATP by mitochondria was not equivalent to $\mathrm{O}_{2}$ consumption in these cells. Hence, we deduced that the cellular redox homeostasis could be altered. Therefore, we tested the production of ROS and found that HuS30gen and HCT116shCTL had increased levels of ROS in comparison to HuS0gen and HCT116shCHK2 (Fig. 7A). Huh7 cells did not show any significant variations in ROS levels. Our findings indicated a significant oxidative stress occurred when the demand of ATP increased. To corroborate these findings, we measured the levels of GSH, the most abundant nonprotein antioxidative stress thiol (30). HuS30gen, HCT116shCTL, and Huh7shCHK2 expressed a significant reduction of GSH as shown in Fig. 7B. Mitochondrial ATP production and mitochondrial 
Lulli et al.
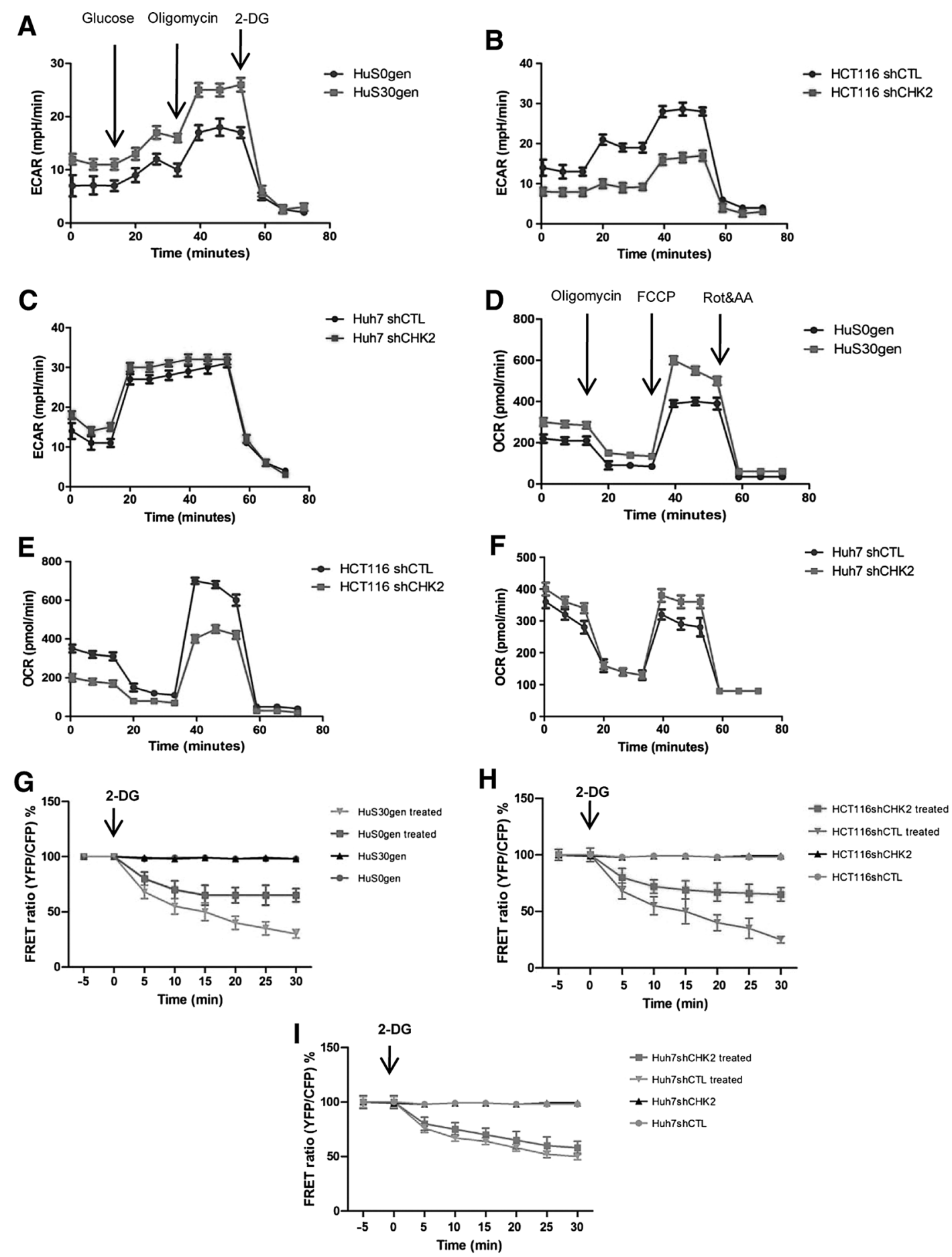

Figure 4.

HuS30gen and HCT116 cells exhibit increased ECAR and OCR and rely on glycolysis the production of ATP. A-C, Seahorse XF glycolysis stress test profile of the key parameters of glycolytic function. In this stress test, cells are exposed sequentially to glucose, oligomycin (ATP synthase inhibitor), and 2-DG (hexokinase inhibitor). D-F, Seahorse XF cell Mito Stress Test profile of the key parameters of mitochondrial respiration. In this stress test, cells are exposed sequentially to oligomycin, FCCP (protonophoric uncoupler), rotenone, and antimycin A (complex I and complex III of electron transport chain inhibitors). Representative graphic of three independent experiments. G-I, Cells expressing ATeam1.03 were generated by transfection with plasmids carrying ATeam $1.03 \mathrm{cDNA}$. The transfected cells were assayed between 1 and 3 days after transfection. Cells expressing ATeam were seeded into $\mu$-dish $35 \mathrm{~mm}$, high glass bottom, in phenol red-free DMEM without glucose, glutamine, and pyruvate. Intracellular ATP depletion in the cells treated with $20 \mathrm{mmol} / \mathrm{L} 2-\mathrm{DG}$ was monitored starting at time $=0$ (min). Cytosolic ATP levels in individual living cells were imaged with ATeam 1.03 using a confocal microscope (see also Supplementary Fig. S5). Sequential confocal images of YFP (top), CFP (middle), and YFP/CFP emission ratio (bottom, pseudocolored) of a HuS30gen cells expressing ATeam1.03. Inhibitor of glycolysis, $20 \mathrm{mmol} / \mathrm{L} 2$-deoxyglucose (2-DG), was added at time 0 (min). Elapsed time (in minutes) after addition of the inhibitor is shown at the top left of the cells. Images were obtained at $37^{\circ} \mathrm{C}(\mathrm{scale}$ bar, $20 \mu \mathrm{m})$. 
A
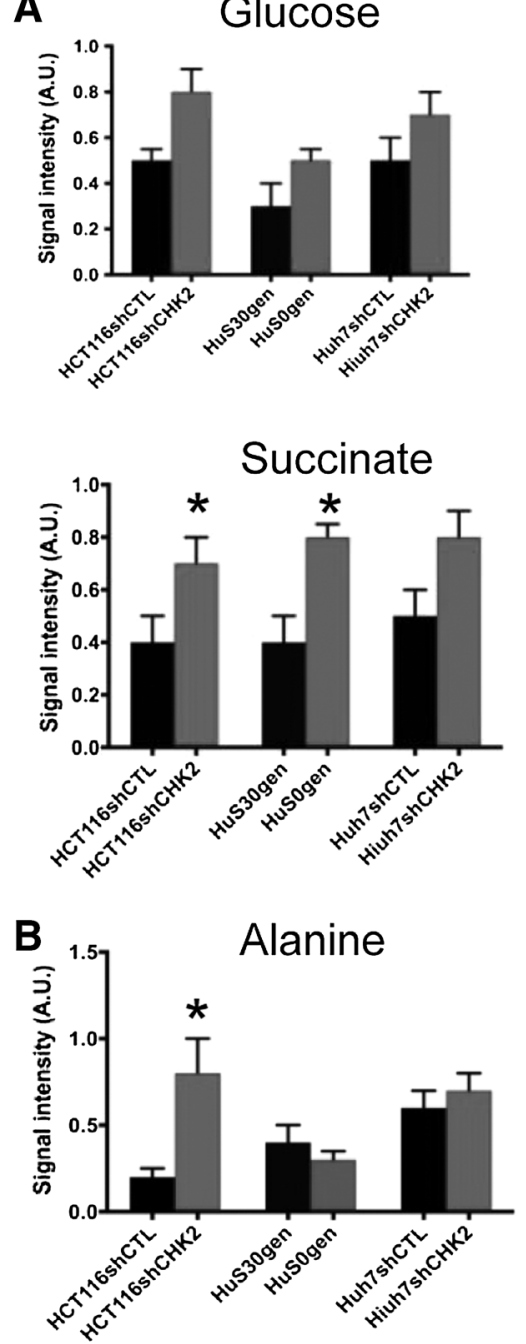

Glutamine

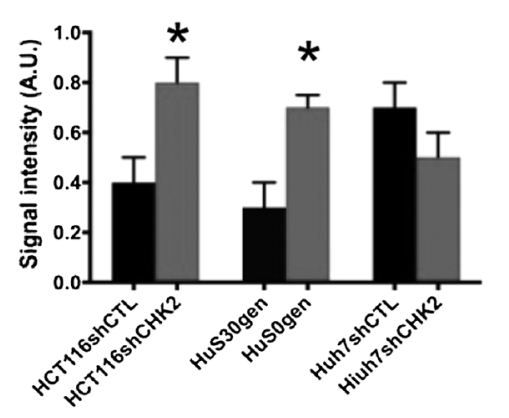

Pyruvate

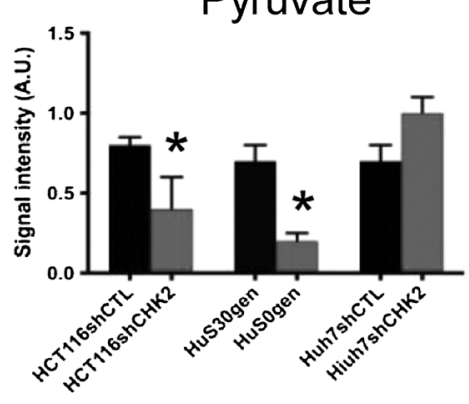

Fumarate

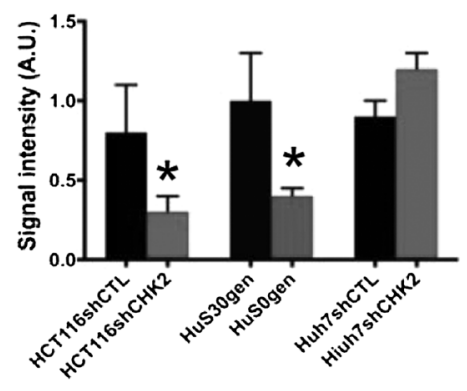

Aspartate

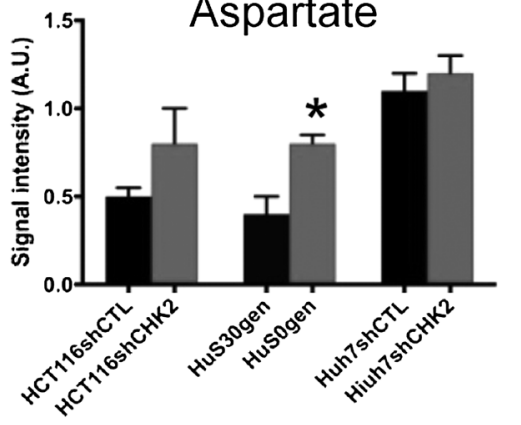

Glycine

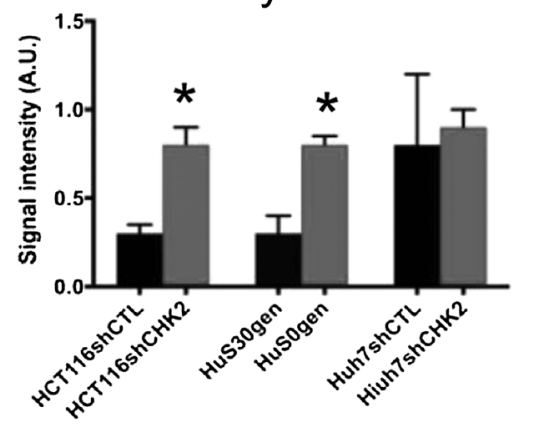

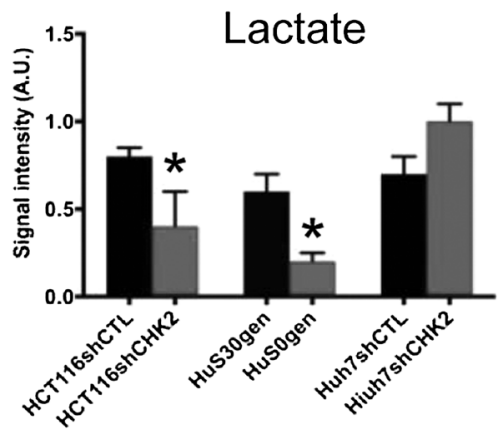

Malate
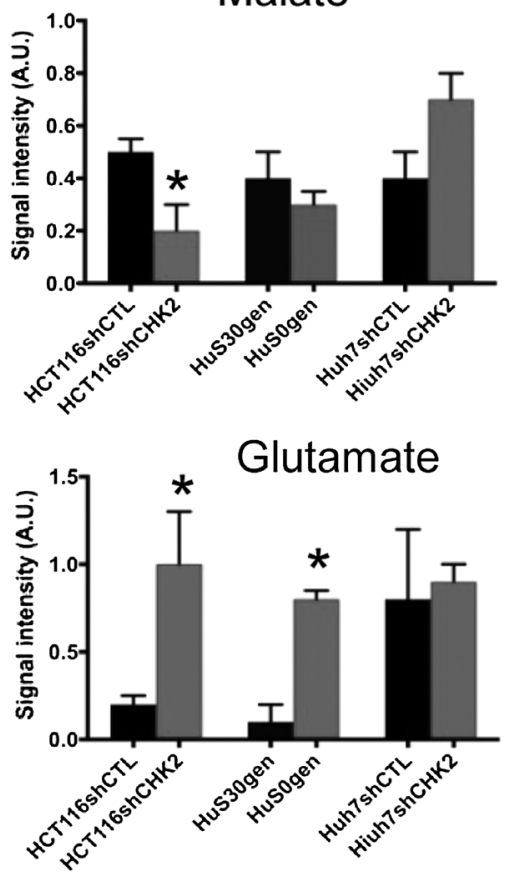

Formate

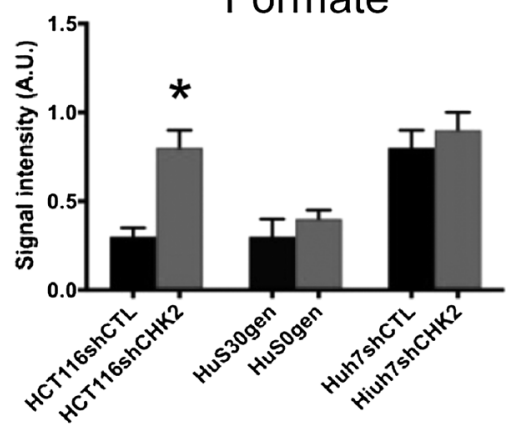

Figure 5.

CHK2 and $\gamma \mathrm{H} 2 \mathrm{AX}$ expression promotes glycolysis and succinate oxidation. A and B, HuSOgen and HuS30gen and CHK2-depleted HCT116 and Huh7 cells were cultured in DMEM with $10 \%$ FBS for 24 hours. ${ }^{1} \mathrm{H}$ NMR spectra were acquired on cell lysates. The relative concentration levels of indicated metabolites calculated by integrating the signal area in the respective ${ }^{1} \mathrm{H}$ NMR spectra are represented as histograms. The experiments were repeated three times. Error bars, mean \pm SD of triplicates. *, $P<0.05$ by unequal variance $t$ test.

membrane potential require $\mathrm{NAD}^{+}$as energy storage. $\mathrm{NAD}^{+}$ gains two electrons and a proton from glycolysis and TCA cycle substrates being reduced to $\mathrm{NADH}$. By measuring $\mathrm{NAD}^{+} / \mathrm{NADH}$ ratio, we found that HuS30gen, HCT116shCTL, and Huh7shCHK2 exhibited a higher $\mathrm{NAD}^{+} / \mathrm{NADH}$ ratio, in comparison with $\mathrm{HuSO}$ gen, HCT116shCHK2, and Huh7shCTL (Fig. 7C). Taken together, these data establish that DDR/CHK2 exert a control on mitochondrial function and increased SDH-driven oxidation provides a 
A
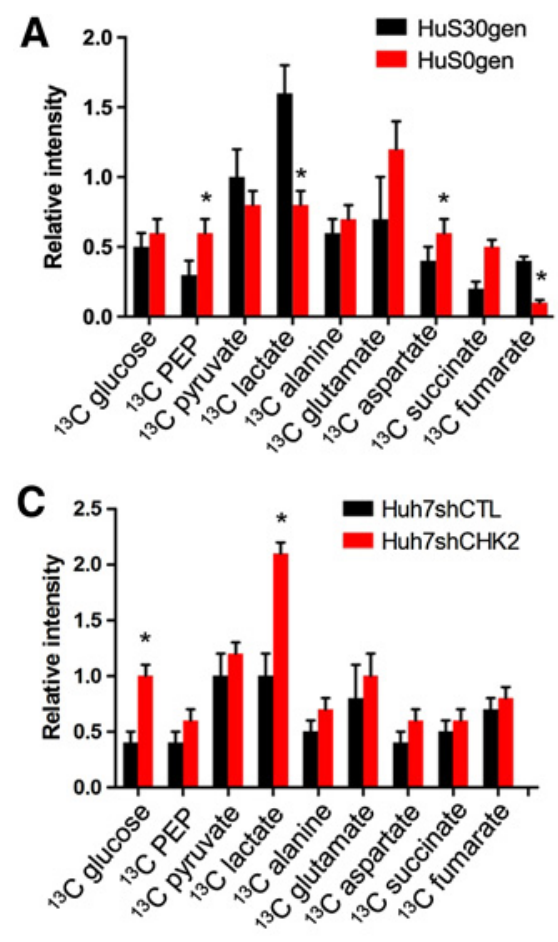

E HCT116shCHK2 vas HCT116shCTL

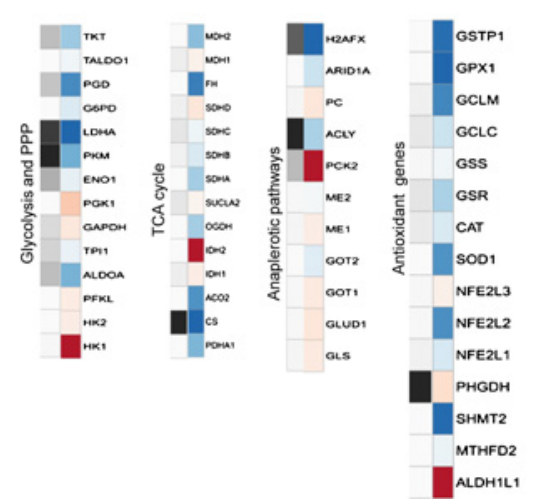

B

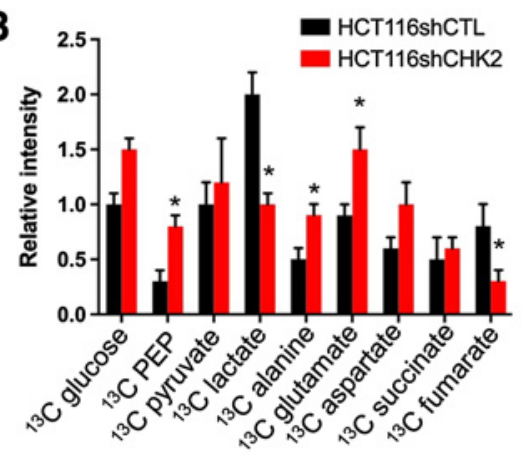

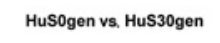
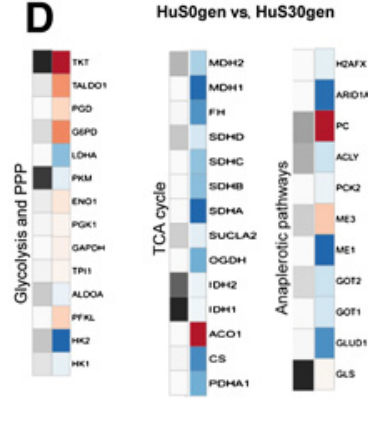

$\mathbf{F}$

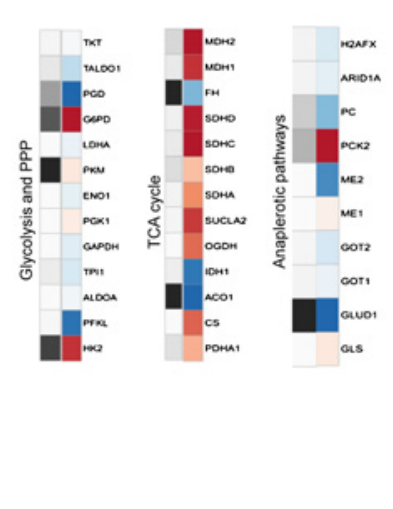

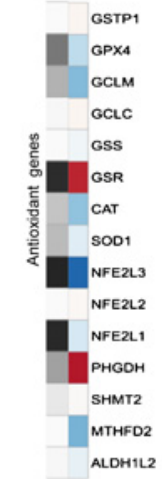

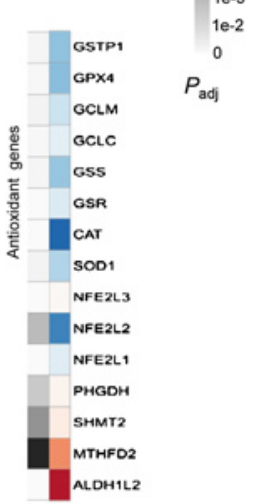

Figure 6.

Metabolomic and transcriptomic evaluations indicate that $\mathrm{CHK} 2$ manages glycolysis and glucose flux through the TCA cycle. A-C, Cells were incubated in DMEM with $10 \mathrm{mmol} / \mathrm{L}$ of $\left[\mathrm{U}^{13} \mathrm{C}_{6}\right]$ glucose for 24 hours and the percentage of enrichment of the indicated metabolites in cell lyates was determined by ${ }^{1} \mathrm{H}-{ }^{13} \mathrm{C}$ NMR as described in Materials and Methods. ${ }^{13} \mathrm{C}$-enriched metabolites levels in CHK2-depleted HCT116 and Huh7 are represented as histograms. ${ }^{13} \mathrm{C}$ enrichment of metabolites was also evaluated in HuSOgen and HuS3Ogen cells. The experiments were repeated three times. Error bars, mean \pm SD of triplicates $\left(^{*}, P<0.05\right.$, by unequal variance $t$ test). D, RNA was isolated and RNA-seq was performed to determine genes significantly downregulated or upregulated in HuS cells. $\mathbf{E}$ and $\mathbf{F}$, RNA was isolated and RNAseq was performed to determine genes significantly downregulated or upregulated in CHK2-depleted HCT116 and Huh7. The strength of the color refers to how strongly upregulated (red) or downregulated (blue) the various genes are. The heat maps colored black and white represent the statistical significance (adjusted $P$ value) of the indicated genes, with the darker colors denoting higher confidence (see also Supplementary Fig. S8). significant contribution toward the high rates of ROS production by mitochondria. Remarkably, CHK2 gene knockdown did reduce mitochondrial production of ROS (30). Furthermore, we address the role of succinate in cell proliferation. Rotenone, a NADHubiquinone oxidoreductase inhibitor, caused a significant reduction of cell proliferation by using $1 \mu \mathrm{mol} / \mathrm{L}$ concentration. Adding diethylsuccinate $(1 \mathrm{mmol} / \mathrm{L})$, a cell-permeable succinate, induced a toxic effect partially attenuated in the HCT116shCHK2 and HuSOgen cells. To confirm the role of SDH, we treated HuS, HCT116, and Huh7 cells with dimethylmalonate (DMM), a potent competitive inhibitor of succinate oxidation by SDH. DMM treatment significantly inhibited the growth of HuS30gen and HCT116shCTL cells, whereas Huh7 cells resulted less sensitive to growth inhibitory effects of DMM (Fig. 7D-F; Supplementary Fig. S9A-S9F). Therefore, we conclude that DDR/CHK2 activation is involved in SDH-dependent ROS production. A high mitochondrial membrane potential $\left(\Delta \psi_{\mathrm{m}}\right)$ is expected in cells demanding elevated ATP production, as in the case of DDR/CHK2 activation. This is because the $\Delta \psi_{\mathrm{m}}$, is generated by NADH oxidation and released $\mathrm{H}^{+}$crossing through complexes I, III, and IV of the mitochondrial inner membrane. Indeed, to determine a potential requirement of $\mathrm{SDH}$ activity for increased $\Delta \psi_{\mathrm{m}}$, we explored the effect of rotenone (Rot), and 2-thenoyltrifluoroacetone (TTFA), a ubiquinone analogue and inhibitor of SDH. The treatment of HuS30gen and HCT116 cells expressing high levels of SDH with Rot increased $\Delta \psi_{\mathrm{m}}$, whereas TTFA significantly reduced $\Delta \psi_{\mathrm{m}}$. The addition of Rot in Huh7 increased the $\Delta \psi_{\mathrm{m}}$, whereas TTFA did not exert any significant reduction of $\Delta \psi_{\mathrm{m}}$ in these cells. Inhibition of $\mathrm{SDH}$ alone or NADH-ubiquinone oxidoreductase $+\mathrm{SDH}$, reduced the mitochondrial TMRM fluorescence at the same extension (Supplementary Fig. S10A-S10E). We, therefore, conclude that SDH effectively plays a key role in sustaining $\Delta \psi_{\mathrm{m}}$ hyperpolarization and ROS production in Rot-treated cells by fueling electron transport to oxygen. 
A

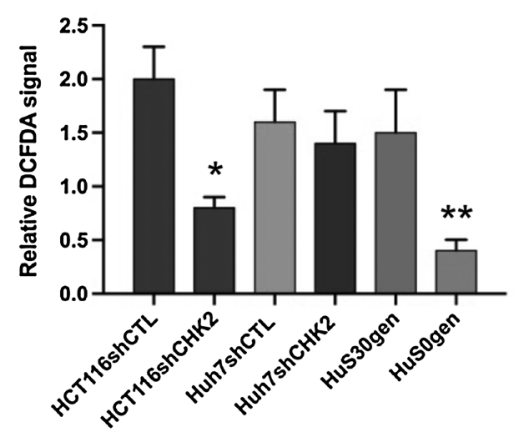

C

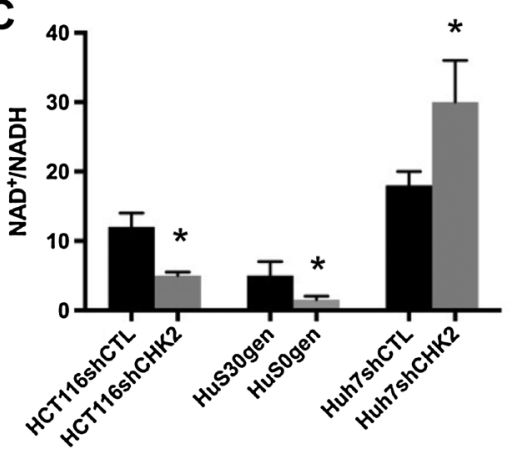

E

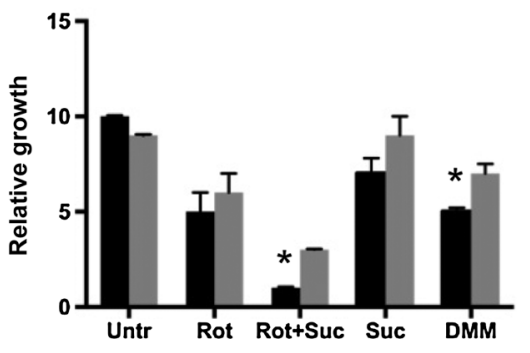

B

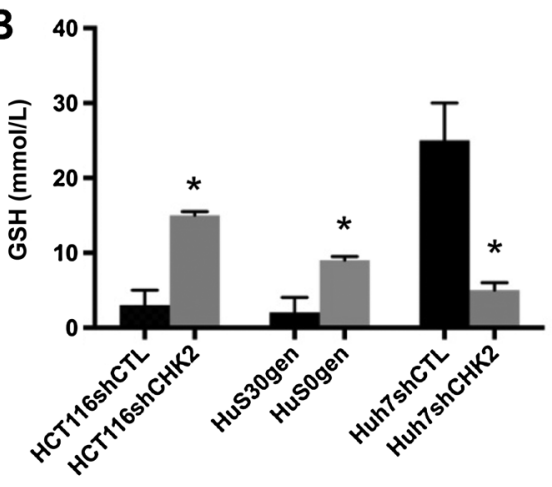

D

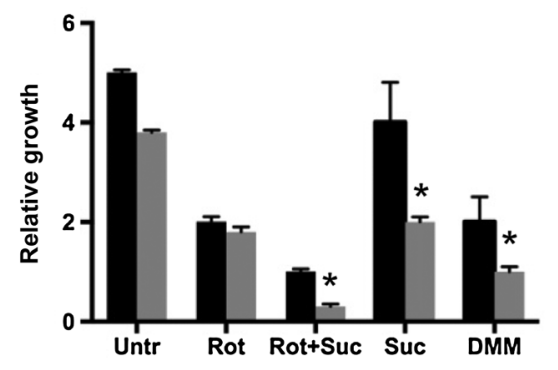

HuSogen

HuS30gen

$\mathbf{F}$

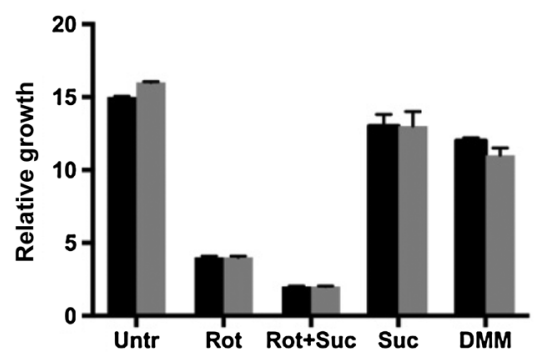

Huh7shCTL

Huh7shCHK2

Figure 7.

The presence of DNA damage/CHK2 promotes ROS production through succinate dehydrogenase activity. A, Relative ROS levels in HuS, HCT116, and Huh7 cells under conditions indicated as determined by DCFDA (29,79-dichlorodihydrofluorescein diacetate) staining. B, GSH levels were measured in lysates of HuSOgen, Hus30gen, HCT116shCTL, and HCT116shCHK2 cells using a colorimetric assay kit. C, NAD ${ }^{+}$NADH ratio measured in lysates of HuSOgen, Hus30gen, HCT116shCTL, HCT116shCHK2, Huh7shCTL, and Huh7shCHK2 using an NAD ${ }^{+} / \mathrm{NADH}$ assay kit. D-F, Proliferation rate was determined in the absence (Untr) or in the presence of cell-permeable diethylsuccinate (Suc, $1 \mathrm{mmol} / \mathrm{L}$ ), NADH oxidoreductase inhibitor rotenone (Rot, $1 \mu \mathrm{mol} / \mathrm{L}$ ), and succinate dehydrogenase inhibitor dimethylmalonate (DMM, $20 \mathrm{mmol} / \mathrm{L}$ ). After 4 days, cells were fixed in $3 \%$ formaldehyde and stained with $0.1 \%$ crystal violet. Dye was extracted with $10 \%$ acetic acid and the relative proliferation was determined by OD at $595 \mathrm{~nm}$ (see also Supplementary Fig. S9). Error bars, mean \pm SD of triplicate wells from five independent experiments. *, $P<0.05$ by two-tailed Student $t$ test.

\section{Discussion}

In this study, we extend the relevance of DNA damage response protein $\mathrm{CHK} 2$ and identify its role in mitochondria functions and glycolysis (31-33). Indeed, our in vivo results indicate that dysplastic/neoplastic hepatocytes, which harbor DNA damage, exhibit high energy demand in terms of glycolysis and oxidative metabolism. Moreover, our data in vitro reveal that cells overexpressing $\gamma-\mathrm{H} 2 \mathrm{AX} / \mathrm{CHK} 2$ own a high oxidative state causing a $\mathrm{SDH}$-dependent ROS production. Furthermore, we provide evi- dence that cells with mitotic-linked DNA damage/CHK2 activation rely on glycolysis for ATP production, and glycolytic ATPproducing enzymes PGK1and PKM2 redistribute from a diffuse localization in the cytoplasm to the mitotic spindle and colocalize with protein CHK2. Therefore, we propose that in conditions of elevated energy demands, as a consequence of mitosis-linked DNA damage, a metabolic compartment is formed within the mitotic spindle to locally implement ATP and accomplish DNA repair and chromosome segregations (34). 
Several studies indicate that dysregulation of mitochondrial functions characterized by TCA cycle disruptions are associated with overproduction of ROS, which may participate in oncogenic signaling and/or tumor progression by modification of DNA (35). We demonstrate that the presence of DNA damage and DDR promotes an enhanced TCA cycle activity, and CHK2 depletion reduces TCA cycle activity. In this situation most amino acids enter the TCA cycle as 4- or 5-carbon compounds, only acetyl-CoA produced from their catabolism can be fully oxidized in the cycle. Therefore, it is important to remove 4-and 5-carbon TCA cycle intermediates to avoid the accumulation in the mitochondrial matrix. These reactions use TCA cycle intermediates as substrate that is converted to a product (e.g., aspartate, glutamate, alanine) that effectively removes intermediates from the cycle (36). In metabolism build-up of metabolites can occur not only due to increased production, but also due to decreased consumption, a process known as cataplerosis (Supplementary Fig. S6C). In addition, we demonstrated that $\mathrm{SDH}$ overexpression funnels succinate oxidation to ROS production. For a long time, SDH has not been considered a relevant contributor to ROS production, however, recently, several studies have established that SDH can produce superoxide anion or $\mathrm{H}_{2} \mathrm{O}_{2}$ at rates similar to complex I and complex III of the ETC $(37,38)$. Our findings support this view with the following observations: (i) the intracellular levels of GSH, the most important antioxidant synthesized in the cells were reduced; (ii) the low levels of cytosolic GSH are directly linked to the overexpression of $\mathrm{CHK} 2 / \mathrm{SDH}$ and ROS, and (iii) CHK2 knockdown restores the cytosolic levels of GSH and reduces ROS production. In this context, ROS could be responsible for creating an imbalance of redox status inducing cells to consume antioxidants such as GSH. Indeed, ROS are essential to cell function, and their role is well established as well as their involvement in many signaling pathways and also in cell growth, but their levels need to be accurately tuned to avoid toxic effects. In this context, $\gamma \mathrm{H} 2 \mathrm{AX}$ may serve as a signal for the timely recruitment and/or retention of DDR proteins in the vicinity of DNA lesions $(39,40)$. Although little is known about the mechanism regulating the exchange of variant histone $\mathrm{H} 2 \mathrm{AX}$ with conventional histone $\mathrm{H} 2 \mathrm{~A}$ in the context of the nucleosome, here we reveal that $\mathrm{CHK} 2$ is involved in $\mathrm{H} 2 \mathrm{AX}$ gene expression $(41,42)$. The central role of mitochondria in DDR signaling is further corroborated by data on formate production $(43,44)$. Recent work has shown that the mitochondrial folate flux exceeds one-carbon

\section{References}

1. Siegel JJ, Amon A. New insights into the troubles of aneuploidy. Annu Rev Cell Dev Biol 2012;28:189-214.

2. Zack TI, Schumacher SE, Carter SL, Cherniack AD, Saksena G, Tabak B, et al. Pan-cancer patterns of somatic copy number alteration. Nat Genet 2013;45: $1134-40$.

3. Sansregret L, Vanhaesebroeck B, Swanton C. Determinants and clinical implications of chromosomal instability in cancer. Nat Rev Clin Oncol 2018;15:139-50.

4. Burrell RA, McClelland SE, Endesfelder D, Groth P, Weller MC, Shaikh N, et al. Replication stress links structural and numerical cancer chromosomal instability. Nature 2013;494:492-6.

5. Davoli T, Uno H, Wooten EC, Elledge SJ. Tumor aneuploidy correlates with markers of immune evasion and with reduced response to immunotherapy. Science 2017;355. DOI: 10.1126/science.aaf8399.

6. Negrini S, Gorgoulis VG, Halazonetis TD. Genomic instability, an evolving hallmark of cancer. Nat Rev Mol Cell Biol 2010;11:220-8.

7. Carloni V, Lulli M, Madiai S, Mello T, Hall A, Luong TV, et al. CHK2 overexpression and mislocalisation within mitotic structures enhances chromosomal instability and hepatocellular carcinoma progression. Gut 2018;67: $348-61$. demand for biosynthesis, producing surplus formate that is excreted from the cell (45). In our study, this pathway seems to be involved supporting the production of glycine to maintain GSH levels to counteract the ROS production elicited by DNA damage/CHK2 activation.

In summary, this study provides the first demonstration of a link between a central effector of DNA damage response such as CHK2 and cellular metabolism. In addition to the increasingly energy demand triggered by DNA damage, activation of CHK2 turns cancer cells into an uncontrollable energetic short circuit. This mechanism can, however, be interrupted by targeting the determinants involved in this process; for example, SDH could be an optimal druggable target to block the energy short circuit required by replication stress-related DNA damage. Furthermore, in this study we have identified a mechanism of ROS production originated by dysfunctional mitochondria. This aspect might represent a vulnerability created by DNA damage response that could be exploited for development of new tools for current and novel therapies.

\section{Authors' Disclosures}

T. Mello reports grants from AIRC - Ente CRF during the conduct of the study. K. Rombouts is a consultant and shareholder of Engitix Therapeutics Ltd. No disclosures were reported by the other authors.

\section{Authors' Contributions}

M. Lulli: Investigation. L. Del Coco: Investigation. T. Mello: Investigation. C. Sukowati: Investigation. S. Madiai: Investigation. L. Gragnani: Investigation. P. Forte: Investigation. F. Fanizzi: Methodology. A. Mazzocca: Investigation. K. Rombouts: Methodology. A. Galli: Writing-review and editing. V. Carloni: Conceptualization, funding acquisition.

\section{Acknowledgments}

This study was supported by Ministero dell' Istruzione, dell' Università e della Ricerca: Prin-bando 2017 (to V. Carloni). This project was supported in part by AIRCEnte CRF with Multi-User Equipment Program no. 19515 (to A. Galli and T. Mello).

The costs of publication of this article were defrayed in part by the payment of page charges. This article must therefore be hereby marked advertisement in accordance with 18 U.S.C. Section 1734 solely to indicate this fact.

Received September 17, 2020; revised February 15, 2021; accepted March 22, 2021; published first March 24, 2021.

8. Tahmasebi-Birgani M, Ansari H, Carloni V. Defective mitosis-linked DNA damage response and chromosomal instability in liver cancer. Biochim Biophys Acta Rev Cancer 2019;1872:60-65.

9. Santaguida S, Amon A. Short- and long-term effects of chromosome missegregation and aneuploidy. Nat Rev Mol Cell Biol 2015;16:473-85.

10. Bakhoum SF, Kabeche L, Compton DA, Powell SN, Bastians H. Mitotic DNA damage response: at the crossroads of structural and numerical cancer chromosome instabilities. Trends Cancer 2017;3:225-34.

11. Chisari FV, Klopchin K, Moriyama T, Pasquinelli C, Dunsford HA, Sell S, et al. Molecular pathogenesis of hepatocellular carcinoma in hepatitis B virus transgenic mice. Cell 1989;59:1145-56.

12. Anfuso B, El-Khobar KE, Ie SI, Avellini C, Radillo O, Raseni A, et al. Activation of hepatic stem cells compartment during hepatocarcinogenesis in a HBsAg HBVtransgenic mouse model. Sci Rep 2018;8:13168.

13. Carloni V, Mazzocca A, Mello T, Galli A, Capaccioli S. Cell fusion promotes chemoresistance in metastatic colon carcinoma. Oncogene 2013;32:2649-60.

14. De Castro F, Benedetti M, Antonaci G, Del Coco L, De Pascali SA, Muscella A, et al. Response of cisplatin resistant Skov-3 cells to $\left[\mathrm{Pt}\left(\mathrm{O}, \mathrm{O}^{\prime}-\mathrm{Acac}\right)(\gamma-\mathrm{Acac})\right.$ (DMS)] treatment revealed by a metabolomic ${ }^{1} \mathrm{H}-\mathrm{NMR}$ study. Molecules 2018 ; 23:2301. 
15. Amiel A, Tremblay-Franco M, Gautier R, Ducheix S, Montagner A., Polizzi A, et al. Proton NMR enables the absolute quantification of aqueous metabolites and lipid classes in unique mouse liver samples. Metabolites 2020;10:9.

16. Gnocchi D, Del Coco L, Girelli CR, Castellaneta F, Cesari G, Sabbà C, et al. ${ }^{1} \mathrm{H}-$ NMR metabolomics reveals a multitarget action of crithmum maritimum ethyl acetate extract in inhibiting hepatocellular carcinoma cell growth. Sci Rep 2021; 11:1-13.

17. Imamura $\mathrm{H}$, Nhat $\mathrm{KP}$, Togawa $\mathrm{H}$, Saito $\mathrm{K}$, Iino $\mathrm{R}$, Kato-Yamada $\mathrm{Y}$, et al. Visualization of ATP levels inside single living cells with fluorescence resonance energy transfer-based genetically encoded indicators. Proc Natl Acad Sci U S A 2009;106:15651-6.

18. Varet H, Brillet-Guéguen L, Coppée JY, Dillies MA. SARTools: A DEseq2- and EdgeR-based R pipeline for comprehensive differential analysis of RNA-seq data. PLoS One 2016;11:e0157022.

19. Love MI, Huber W, Anders S. Moderated estimation of fold change and dispersion for RNA-seq data with DESeq2. Genome Biol 2014;15:550.

20. Gao R, Cheng J, Fan C, Shi X, Cao Y, Sun B, et al. Serum metabolomics to identify the liver disease-specific biomarkers for the progression of hepatitis to hepatocellular carcinoma. Sci Rep 2015;5:18175.

21. Israelsen WJ, Vander Heiden MG. Pyruvate kinase: function, regulation and role in cancer. Semin Cell Dev Biol 2015;43:43-51.

22. Christofk HR, Vander Heiden MG, Harris MH, Ramanathan A, Gerszten RE Wei R, et al. The M2 splice isoform of pyruvate kinase is important for cancer metabolism and tumour growth. Nature 2008;452:230-3.

23. King A, Selak MA, Gottlieb E. Succinate dehydrogenase and fumarate hydratase: linking mitochondrial dysfunction and cancer. Oncogene 2006;25:4675-82.

24. Gorgoulis VG, Vassiliou LV, Karakaidos P, Zacharatos P, Kotsinas A, Liloglou T et al. Activation of the DNA damage checkpoint and genomic instability in human precancerous lesions. Nature 2005;434:907-13.

25. Rogakou EP, Pilch DR, Orr AH, Ivanova VS, Bonner WM. DNA doublestranded breaks induce histone $\mathrm{H} 2 \mathrm{AX}$ phosphorylation on serine 139. J Biol Chem 1998;273:5858-68.

26. Kruhlak MJ, Celeste A, Dellaire G, Fernandez-Capetillo O, Müller WG, McNally JG, et al. Changes in chromatin structure and mobility in living cells at sites of DNA double-strand breaks. J Cell Biol 2006;172:823-34.

27. Hu H, Zhu W, Qin J, Chen M, Gong L, Li L, et al. Acetylation of PGK1 promotes liver cancer cell proliferation and tumorigenesis. Hepatology 2017;65:515-28.

28. Vander Heiden MG, DeBerardinis RJ. Understanding the intersections between metabolism and cancer biology. Cell 2017;168:657-69.

29. Sheltzer JM. A transcriptional and metabolic signature of primary aneuploidy is present in chromosomally unstable cancer cells and informs clinical prognosis. Cancer Res 2013;73:6401-12.
30. Liemburg-Apers DC, Willems PH, Koopman WJ, Grefte S. Interactions between mitochondrial reactive oxygen species and cellular glucose metabolism. Arch Toxicol 2015;89:1209-26.

31. Crasta K, Ganem NJ, Dagher R, Lantermann AB, Ivanova EV, Pan Y, et al. DNA breaks and chromosome pulverization from error in mitosis. Nature 2012;482: 53-58.

32. Polo SE, Jackson SP. Dynamics of DNA damage response proteins at DNA breaks: a focus on protein modifications. Genes Dev 2011;25:409-33.

33. Halazonetis TD, Gorgoulis VG, Bartek J. An oncogene-induced DNA damage model for cancer development. Science 2008;319:1352-5.

34. Jang S, Nelson JC, Bend EG, Rodríguez-Laureano L, Tueros FG, Cartagenova L et al. Glycolytic enzymes localize to synapses under energy stress to support synaptic function. Neuron 2016;90:278-91.

35. Yang M, Soga T, Pollard PJ. Oncometabolites: linking altered metabolism with cancer. J Clin Invest 2013;123:3652-8.

36. Owen OE, Kalhan SC, Hanson RW. The key role of anaplerosis and cataplerosis for citric acid cycle function. J Biol Chem 2002;277:30409-12.

37. Moreno-Sánchez R, Hernández-Esquivel L, Rivero-Segura NA, Marín-Hernán$\operatorname{dez}$ A, Neuzil J, Ralph SJ, et al. Reactive oxygen species are generated by the respiratory complex II evidence for lack of contribution of the reverse electron flow in complex I. FEBS J 2013;280:927-38.

38. Quinlan CL, Orr AL, Perevoshchikova IV, Treberg JR, Ackrell BA, Brand MD Mitochondrial complex II can generate reactive oxygen species at high rates in both the forward and reverse reactions. J Biol Chem 2012;287:27255-64.

39. Tanaka T, Halicka HD, Huang X, Traganos F, Darzynkiewicz Z. Constitutive histone $\mathrm{H} 2 \mathrm{AX}$ phosphorylation and ATM activation, the reporters of DNA damage by endogenous oxidants. Cell Cycle 2006;5:1940-5.

40. Guo Z, Kozlov S, Lavin MF, Person MD, Paull TT. ATM activation by oxidative stress. Science 2010;330:517-21.

41. Bonner WM, Redon CE, Dickey JS, Nakamura AJ, Sedelnikova OA, Solier S, et al. $\gamma$-H2AX and cancer. Nat Rev Cancer 2008;8:957-67.

42. Heo K, Kim H, Choi SH, Choi J, Kim K, Gu J, et al. FACT-mediated exchange of histone variant $\mathrm{H} 2 \mathrm{AX}$ regulated by phosphorylation of $\mathrm{H} 2 \mathrm{AX}$ and ADPribosylation of Spt16. Mol Cell 2008;30:86-97.

43. Yang M, Vousden K. Serine and one-carbon metabolism in cancer. Nat Rev Cancer 2016;16:650-62.

44. Ye J, Fan J, Venneti S, Wan YW, Pawel BR, Zhang J, et al. Serine catabolism regulates mitochondrial redox control during hypoxia. Cancer Discov 2014;4: 1406-17.

45. Meiser J, Schuster A, Pietzke M, VandeVoorde J, Athineos D, Oizel K, et al. Increased formate overflow is a hallmark of oxidative cancer. Nat Commun 2018; 9:1368. 


\section{Cancer Research}

$A A C R$ 年erican Assciation

The Journal of Cancer Research (1916-1930) | The American Journal of Cancer (1931-1940)

\section{DNA Damage Response Protein CHK2 Regulates Metabolism in Liver Cancer}

Matteo Lulli, Laura Del Coco, Tommaso Mello, et al.

Cancer Res 2021;81:2861-2873. Published OnlineFirst March 24, 2021.

Updated version Access the most recent version of this article at: doi:10.1158/0008-5472.CAN-20-3134

Supplementary Access the most recent supplemental material at:

Material http://cancerres.aacrjournals.org/content/suppl/2021/03/24/0008-5472.CAN-20-3134.DC1

Cited articles This article cites 44 articles, 11 of which you can access for free at:

http://cancerres.aacrjournals.org/content/81/11/2861.full\#ref-list-1

E-mail alerts Sign up to receive free email-alerts related to this article or journal.

Reprints and To order reprints of this article or to subscribe to the journal, contact the AACR Publications Department at Subscriptions pubs@aacr.org.

Permissions To request permission to re-use all or part of this article, use this link http://cancerres.aacrjournals.org/content/81/11/2861.

Click on "Request Permissions" which will take you to the Copyright Clearance Center's (CCC) Rightslink site. 